\title{
OPEN A recombinase polymerase amplification assay for rapid detection of rabies virus
}

\author{
Martin Faye ${ }^{1 \bowtie}$, Ahmed Abd El Wahed ${ }^{2,3}$, Oumar Faye ${ }^{1}$, Jonas Kissenkötter ${ }^{2}$, \\ Bernd Hoffmann ${ }^{4}$, Amadou Alpha Sall ${ }^{1}$ \& Ousmane Faye ${ }^{1}$
}

Rabies is a generally fatal encephalitis caused by a negative-sense single-stranded RNA lyssavirus transmitted to humans mainly from dog bite. Despite the recommendation by WHO and OIE to use the direct immunofluorescence test as standard method, molecular diagnostic assays like reverse transcription quantitative polymerase chain reaction (RT-qPCR) are increasing as a confirmatory method. However, both technologies are inaccessible in resource-limited settings. Moreover, the available point-of-need molecular assay is of poor detection limit for African strains. Herein, we developed a reverse transcription recombinase polymerase amplification (RT-RPA) assay as potential point-of-need diagnostic tool for rapid detection of various strains of rabies virus including locally isolated African strains. The sensitivity and specificity of the method was evaluated using a molecular RNA standard and different Rabies-related viruses belonging to the Rhabdoviridea family, respectively. The RABV-RPA performances were evaluated on isolates representative of the existing diversity and viral dilutions spiked in non-neural clinical specimen. The results were compared with RT-qPCR as a gold standard. The RABV-RPA detected down to 4 RNA molecules per reaction in $95 \%$ of the cases in less than $10 \mathrm{~min}$. The RABV-RPA assay is highly specific as various RABV isolates were identified, but no amplification was observed for other member of the Rhabdoviridea family. The sample background did not affect the performance of the RABV-RPA as down to 11 RNA molecules were identified, which is similar to the RT-qPCR results. Our developed assay is suitable for use in low-resource settings as a promising alternative tool for ante-mortem rabies diagnosis in humans for facilitating timely control decisions.

Rabies virus (RABV) is an enveloped pathogen belonging to the Lyssavirus genus (order Mononegavirales, family Rhabdoviridae) and its genome is a negative single-stranded RNA of approximately 12 kilobases (kb) in size encoding five proteins (N, P, M, G, L) separated by four intergenic regions and encompassed between a leader region and a trailer ( $3^{\prime}$ and $5^{\prime}$ non-coding regions) ${ }^{1}$. The RABV presents a large genetic diversity worldwide, with particularly four phylogenetic groups circulating in Africa ${ }^{2,3}$. It is responsible of a neglected zoonotic disease affecting several mammals in many parts of the world and humans are generally exposed through dog bites ${ }^{4,5}$. However, Rabies transmission was also reported through transplantation of organs from undiagnosed donors with a long incubation period of the virus $s^{6,7}$. Rabies infection is fatal in most of cases with an annual death toll of 60,000 in humans worldwide ${ }^{1}$.

The highest public health impact of Rabies is recorded in Asian and African developing countries ${ }^{4,8}$ and $40 \%$ of deaths occur in children under 15 years-old ${ }^{5,9}$. Nevertheless, early detection of cases is crucial to provide rapid outbreak response and emergency vaccination measures. Several methods have been previously described for the diagnosis of rabies virus (RABV) infection including direct virus isolation ${ }^{10}$, immunohistochemistry ${ }^{10-12}$, immunochromatography ${ }^{13,14}$ and immunofluorescence ${ }^{15,16}$, as well as sensitive molecular methods such as realtime reverse transcription polymerase chain reaction (RT-PCR) assay ${ }^{17-22}$. However, development of reliable broad spectrum and economical tests remains a challenge to improve surveillance of rabies cases in the field to achieve goal \#3 of the sustainable development goals $s^{17,20,23}$.

Recombinase polymerase amplification (RPA) is an isothermal molecular tool with portable instrumentation currently used in many diagnostic fields as an alternative to the PCR ${ }^{24,25}$. RPA is becoming a molecular tool of

${ }^{1}$ Virology Department, Institut Pasteur de Dakar, 36, Avenue Pasteur, 220 Dakar, Senegal. ${ }^{2}$ Virology Lab, Division of Microbiology and Animal Hygiene, University of Göttingen, Göttingen, Germany. ${ }^{3}$ Institute of Animal Hygiene and Veterinary Public Health, University of Leipzig, Leipzig, Germany. ${ }^{4}$ Institute of Diagnostic Virology, Friedrich-L oeffler-Institute, Greifswald-Insel Riems, Germany. ${ }^{\bowtie}$ email: martin.faye@pasteur.sn 
A)

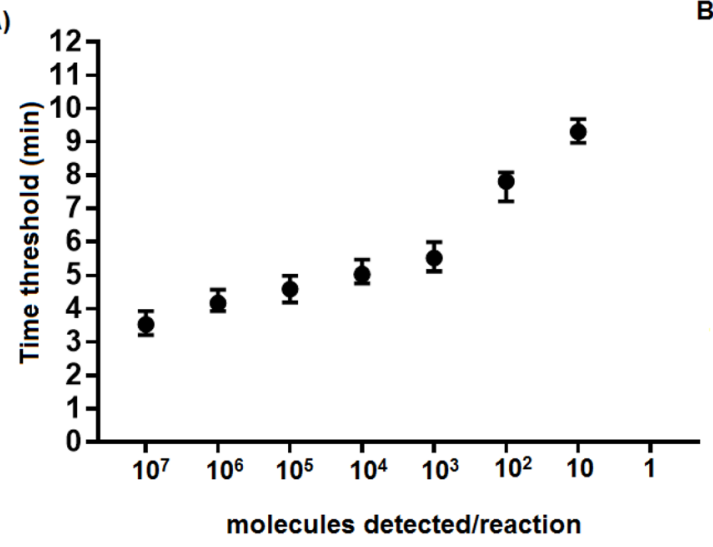

B)

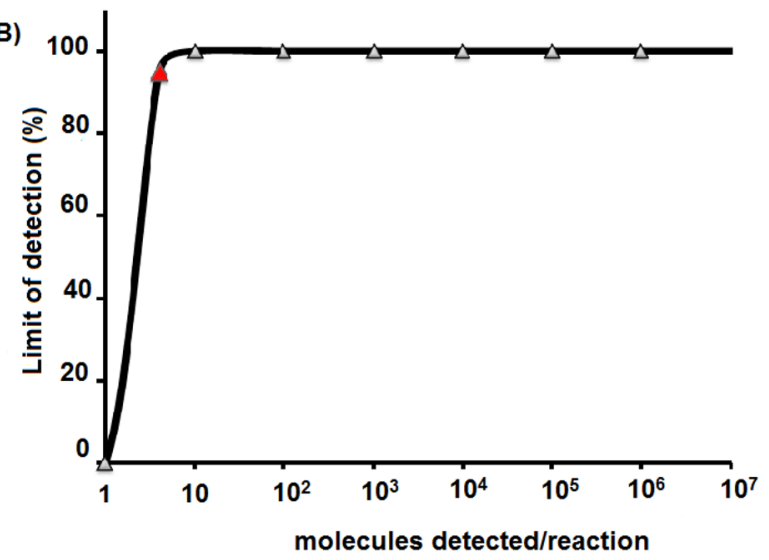

Figure 1. Analytical sensitivity of the RABV-RPA assay. A semi-log regression analysis (A) was performed by plotting the RABV-RPA threshold time values against the number of RNA molecules detected in eight replicates (8/8). The RABV-RPA assay produced positive results between 3 and $10 \mathrm{~min}$. Dilutions $10^{7}$ to 10 RNA molecules were detected 8 out of 8 runs and 1 molecule was not identified by the RT-RPA assay. The dots represent the mean values and the error bars represent the standard deviation. The probit regression analysis (B) was performed using data of eight RABV-RPA assay runs. The limit of detection at 95\% probability (4 RNA molecules/reaction) is depicted by the red triangle. The graphs were plotted using PRISM (Graphpad Software Inc., San Diego, California).

choice for the rapid, specific, and cost-effective identification of pathogens. RPA is integrated in point-of-care (POC) bioassays, suitcase lab and on handheld automated fluidic platform $\mathrm{s}^{26,27}$. The method rely on a bacterial recombinase enzyme to anneal primers to template and isothermal DNA polymerase for the DNA amplification step. A reverse transcription RPA (RT-RPA) platform utilized both reverse Transcriptase Moloney Murine Leukemia Virus for the RT step and a fluorescent probe system for real-time data acquisition ${ }^{28-30}$. In the past decade, RPA technology was widely used for molecular diagnosis of pathogens of public health concern ${ }^{31-38}$. Because of its simplicity (few and easy hands-on steps), flexibility (large range of commercial kits available in dried formats), completely isothermal (i.e. no need to perform an enzyme pre-activation) and low-temperature profile $\left(37-42^{\circ} \mathrm{C}\right.$ ) and speed (results in 5-20 min), RPA technology has been successfully used for rapid detection of various pathogens without requirement of any sophisticated equipment ${ }^{25,39}$. RT-RPA for the rapid detection of RABV was previously developed (Schlottau assay) ${ }^{21}$, but we have discovered that it is not suitable for African RABV strains.

In this study, a rapid and sensitive fluorescent probe-based RT-RPA assay was developed and evaluated for rapid and broad range detection of Rabies virus (RABV). Limit of detection was measured with a synthetized standard RNA. Diagnostic accuracy was determined with collection of positive and negative specimen from various virus strains. Finally, clinical performance was checked using cerebro-spinal fluid spiked with RABV.

\section{Results}

Primers selection. A total of 4 forward primers (FPs), 4 reverse primers (RPs), and one exo probe targeting the conserved region of the nucleocapsid gene $(\mathrm{N})$ were initially designed. The 16 combinations were screened with the $10^{5}$ RNA molecules/reaction of the in vitro transcribed RNA standard. The combination with the highest and earliest start of exponential amplification curve was selected and tested with tenfold serial dilutions (from $10^{5}$ to 10 RNA molecules/reaction) of the standard RNA. Unfortunately, the selected primers pair (FP2/ RP1) enabled detection down to $10^{3}$ RNA molecules/reaction, which was not enough. Therefore, many primers were designed in order to select the one that produced the most sensitive assay. In total, 179 additional combination were screened. The primer pair (RPA_N_FP2C/RPA_N_RP4C3P) enabled detection down to 10 molecule/ reaction of the molecular RNA standard.

Analytical sensitivity. The analytical sensitivity was determined with Threshold time (Tt) data values from eight sets of tenfold dilutions of the molecular standard RNA ranging from $10^{7}$ to 1 molecules/reaction. As low as 10 molecules/reaction within $10 \mathrm{~min}$ was detected in the new RABV RT-RPA (Fig. 1A). The probit analysis revealed the limit of detection in 95\% of cases was 4 RNA molecules/reaction (Fig. 1B). Intra-run and inter-run coefficients of variation (CVs) were calculated from eight Tt data values of the dilution $10^{5}$ standard RNA molecules/reaction tested in the same run and in eight different runs. Intra-run and inter-run (CVs) were $0.54 \%$ and $3.40 \%$ for the RABV-RPA assay, respectively.

Analytical specificity. All RABV strains were detected while no cross-reaction was observed with rabiesrelated virus species belonging to the Rhadoviridae family, showing that the RABV-RPA assay is highly specific for RABV detection (Table 1). To confirm the specificity of the developed RT-RPA assay, five RPA amplicons from RABV isolates belonging to distant clades were purified and sequenced using the Illumina Miseq platform (Illumina, San Diego, CA, USA). The BLAST analysis (https://blast.ncbi.nlm.nih.gov/) of obtained sequences showed 


\begin{tabular}{|c|c|c|c|c|c|c|c|c|c|c|}
\hline \multirow[b]{2}{*}{ Isolates } & \multirow[b]{2}{*}{ Virus } & \multirow[b]{2}{*}{ Genus } & \multirow[b]{2}{*}{ Reference } & \multirow{2}{*}{$\begin{array}{l}\text { Place of } \\
\text { isolation }\end{array}$} & \multirow[b]{2}{*}{ Year of isolation } & \multirow[b]{2}{*}{ Species } & \multicolumn{2}{|c|}{ Rabies virus RT-qPCR } & \multicolumn{2}{|l|}{ RABV-RPA } \\
\hline & & & & & & & Mean Cq value ${ }^{\mathrm{a}}$ & SD & Mean Tt value ${ }^{b}$ & SD \\
\hline SA221203SEN & Rabies (RABV) & Lyssavirus & NRC-rabies IPD & Senegal & 2011 & Mellivora capensi & 24.07 & 0.18 & 2.53 & 0.01 \\
\hline SA217694SEN & Rabies (RABV) & Lyssavirus & NRC-rabies IPD & Senegal & 2011 & \begin{tabular}{|l} 
Canis lupus \\
familiaris
\end{tabular} & 22.41 & 0.28 & 5.97 & 0.12 \\
\hline SA217695SEN & Rabies (RABV) & Lyssavirus & NRC-rabies IPD & Senegal & 2011 & $\begin{array}{l}\text { Canis lupus } \\
\text { familiaris }\end{array}$ & 27.44 & 0.07 & 4.81 & 0.02 \\
\hline SA217750SEN & Rabies (RABV) & Lyssavirus & NRC-rabies IPD & Senegal & 2011 & $\begin{array}{l}\text { Canis lupus } \\
\text { familiaris }\end{array}$ & 24.99 & 0.20 & 2.70 & 0.06 \\
\hline SH218152SEN & Rabies (RABV) & Lyssavirus & NRC-rabies IPD & Senegal & 2011 & Homo sapiens & 18.82 & 0.06 & 2.26 & 0.25 \\
\hline SH177846SEN & Rabies (RABV) & Lyssavirus & NRC-rabies IPD & Senegal & 2005 & Homo sapiens & 18.88 & 0.04 & 2.46 & 0.01 \\
\hline SA194858SEN & Rabies (RABV) & Lyssavirus & NRC-rabies IPD & Senegal & 2008 & \begin{tabular}{|l} 
Canis lupus \\
familiaris
\end{tabular} & 26.65 & 0.23 & 3.36 & 0.05 \\
\hline SA204014SEN & Rabies (RABV) & Lyssavirus & NRC-rabies IPD & Senegal & 2010 & $\begin{array}{l}\text { Canis lupus } \\
\text { familiaris }\end{array}$ & 19.43 & 0.34 & 2.37 & 0.01 \\
\hline SA206776SEN & Rabies (RABV) & Lyssavirus & NRC-rabies IPD & Senegal & 2010 & $\begin{array}{l}\text { Canis lupus } \\
\text { familiaris }\end{array}$ & 19.12 & 0.08 & 2.03 & 0.08 \\
\hline SA252888SEN & Rabies (RABV) & Lyssavirus & NRC-rabies IPD & Senegal & 2013 & $\begin{array}{l}\text { Canis lupus } \\
\text { familiaris }\end{array}$ & 18.48 & 0.08 & 2.25 & 0.04 \\
\hline SA252913SEN & Rabies (RABV) & Lyssavirus & NRC-rabies IPD & Senegal & 2013 & $\begin{array}{l}\text { Canis lupus } \\
\text { familiaris }\end{array}$ & 21.90 & 0.08 & 2.48 & 0.14 \\
\hline SA262037SEN & Rabies (RABV) & Lyssavirus & NRC-rabies IPD & Senegal & 2013 & $\begin{array}{l}\text { Canis lupus } \\
\text { familiaris }\end{array}$ & 20.06 & 0.04 & 2.51 & 0.09 \\
\hline SA262503SEN & Rabies (RABV) & Lyssavirus & NRC-rabies IPD & Senegal & 2014 & $\begin{array}{l}\text { Canis lupus } \\
\text { familiaris }\end{array}$ & 21.13 & 0.04 & 2.44 & 0.21 \\
\hline SA262518SEN & Rabies (RABV) & Lyssavirus & NRC-rabies IPD & Senegal & 2014 & $\begin{array}{l}\text { Canis lupus } \\
\text { familiaris }\end{array}$ & 20.38 & 0.06 & 2.35 & 0.11 \\
\hline SA267115SEN & Rabies (RABV) & Lyssavirus & NRC-rabies IPD & Senegal & 2014 & $\begin{array}{l}\text { Canis lupus } \\
\text { familiaris }\end{array}$ & 19.48 & 0.13 & 2.29 & 0.15 \\
\hline DakAnB1094 & Kolongo (KOLV) & unassigned & \begin{tabular}{|l|} 
JX276998 \\
\end{tabular} & CAR & 1970 & Euplectes afra & $\mathrm{Neg}$ & Neg & Neg & Neg \\
\hline AnY1307 & \begin{tabular}{|l|}
$\begin{array}{l}\text { Mokola } \\
(\text { MOKV) }\end{array}$ \\
\end{tabular} & Lyssavirus & NC_006429 & Cameroon & 1973 & Crocidura spp. & Neg & Neg & Neg & Neg \\
\hline AnB373d & $\begin{array}{l}\begin{array}{l}\text { Sandjimba } \\
\text { (SJAV) }\end{array} \\
\end{array}$ & unassigned & JX277024 & CAR & 1970 & \begin{tabular}{|l|}
$\begin{array}{l}\text { Acrocephalus } \\
\text { schoenobaenus }\end{array}$ \\
\end{tabular} & Neg & Neg & Neg & Neg \\
\hline AnB4289 & Nasoule (NASV) & unassigned & JX277012 & CAR & 1973 & \begin{tabular}{|l|} 
Andropadus \\
virens
\end{tabular} & Neg & Neg & Neg & Neg \\
\hline AnD42443 & $\begin{array}{l}\text { Lagos Bat } \\
\text { (LBV) } 3\end{array}$ & Lyssavirus & NC020807 & Senegal & 1985 & Eidolon helvum & Neg & Neg & $\mathrm{Neg}$ & Neg \\
\hline AnB672 & $\begin{array}{l}\text { Lagos Bat } \\
\text { (LBV) } 2\end{array}$ & Lyssavirus & NRC-rabies IPD & CAR & 1974 & $\begin{array}{l}\text { Micropteropus } \\
\text { pusillus }\end{array}$ & Neg & Neg & Neg & Neg \\
\hline An K 6909 & $\begin{array}{l}\text { Lagos Bat } \\
\text { (LBV) } 4\end{array}$ & Lyssavirus & NRC-rabies IPD & Guinea & 1985 & $\begin{array}{l}\text { Nycteris gam- } \\
\text { biensis }\end{array}$ & Neg & Neg & Neg & Neg \\
\hline LBVNIG1956 & $\begin{array}{l}\text { Lagos Bat } \\
\text { (LBV) } 6\end{array}$ & Lyssavirus & EF547431 & Nigeria & 1956 & Eidolon helvum & Neg & Neg & Neg & Neg \\
\hline DakHD763 & $\begin{array}{l}\text { Le Dantec } \\
\text { (LDV) }\end{array}$ & Ledantevirus & AY854650 & Senegal & 1965 & Homo sapiens & Neg & Neg & $\mathrm{Neg}$ & Neg \\
\hline DakAnD5314 & $\begin{array}{l}\text { Keuraliba } \\
\text { (KEUV) }\end{array}$ & Ledantevirus & JX276996 & Senegal & 1968 & Tatera kempi & Neg & Neg & $\mathrm{Neg}$ & Neg \\
\hline ArD89384 & $\begin{array}{l}\text { Chandipura } \\
\text { (CHNV) }\end{array}$ & Vesiculovirus & NRC-rabies IPD & Senegal & 1992 & Phlebotomus sp. & Neg & Neg & Neg & Neg \\
\hline SudAr1275 & \begin{tabular}{|l}
$\begin{array}{l}\text { Obodhiang } \\
\text { (OBOV) }\end{array}$ \\
\end{tabular} & Ephemerovirus & HM856902 & Sudan & 1963 & $\begin{array}{l}\text { Mansonia } \\
\text { uniformis }\end{array}$ & Neg & Neg & Neg & Neg \\
\hline ArY31-65 & $\begin{array}{l}\text { Nkolbisson } \\
\text { (NKOV) }\end{array}$ & Ledantevirus & JX277015 & Cameroon & 1965 & \begin{tabular}{|l} 
Eretmapodites \\
leucopus
\end{tabular} & Neg & Neg & Neg & Neg \\
\hline DakAnB439 & Garba (GARV) & unassigned & KM204982 & CAR & 1970 & $\begin{array}{l}\text { Corythornis } \\
\text { cristata }\end{array}$ & Neg & Neg & Neg & Neg \\
\hline SAAr1995 & $\begin{array}{l}\text { Mossuril } \\
\text { (MOSV) }\end{array}$ & Hapavirus & KM204993 & Mozambique & 1959 & Culex sitiens & Neg & Neg & Neg & Neg \\
\hline
\end{tabular}

Table 1. Description and results of rhabdoviruses isolates used in this study for specificity assessment. Tt: time threshold in minutes, CAR: Central African Republic, SD: standard deviation, Neg: negative; no crossreactivity, Cq: quantitative cycle, NRC-Rabies IPD: national reference center for rabies virus (NRC-Rabies) at Institut Pasteur de Dakar, BP 220, Dakar, Senegal. ${ }^{a}$ Mean Cq value from duplicates with the previously described RABV-L-protein real-time RT-qPCR assay by Faye et al. used as reference test ${ }^{19}$. ${ }^{\mathrm{b}} \mathrm{Mean} \mathrm{Tt}$ value from duplicates given by the new established rabies virus RT-RPA assay. 


\begin{tabular}{|c|c|c|c|c|c|c|c|c|}
\hline \multirow[b]{2}{*}{ Isolates } & \multirow{2}{*}{$\begin{array}{l}\text { Phylogenetic clade- } \\
\text { subclade }\end{array}$} & \multirow[b]{2}{*}{ Reference } & \multirow[b]{2}{*}{ Place of isolation } & \multirow[b]{2}{*}{ Year of isolation } & \multirow[b]{2}{*}{ Species } & \multirow{2}{*}{$\begin{array}{l}\text { Rabies virus } \\
\text { RT-qPCR } \\
\text { Mean Cq value }^{\mathrm{a}}\end{array}$} & \multirow{2}{*}{$\begin{array}{l}\text { RABV-RPA } \\
\text { Mean Tt value }^{\mathrm{b}}\end{array}$} & \multirow{2}{*}{$\begin{array}{l}\text { Schlottau assay } \\
\text { Mean Tt value }\end{array}$} \\
\hline & & & & & & & & \\
\hline SH155966SEN & Africa 2 & NRC-rabies IPD & Senegal & 2001 & Human & 24.67 & 3.23 & 4.94 \\
\hline SA267333SEN & Africa 2 & NRC-rabies IPD & Senegal & 2014 & Dog & 25.36 & 5.58 & $\mathrm{Neg}$ \\
\hline SA173837SEN & Africa 2 & NRC-rabies IPD & Senegal & 2004 & Dog & 28.52 & 5.24 & $\mathrm{Neg}$ \\
\hline SH189343SEN & Africa 2 & NRC-rabies IPD & Senegal & 2007 & Human & 18.32 & 2.46 & 7.77 \\
\hline SA272282SEN & Africa 2 & NRC-rabies IPD & Senegal & 2015 & Dog & 18.29 & 1.87 & 5.23 \\
\hline SH290289SEN & Africa 2 & NRC-rabies IPD & Senegal & 2017 & Human & 22.69 & 2.92 & 7.17 \\
\hline SH290371SEN & Africa 2 & NRC-rabies IPD & Senegal & 2017 & Human & 23.10 & 5.00 & $\mathrm{Neg}$ \\
\hline 91047FRA & Cosmopolitan-WE & KX148127 & France & 1991 & Fox & 20.41 & 6.88 & 6.71 \\
\hline 96140POL & Cosmopolitan-CE & KX148120 & Poland & 1993 & Raccoon dog & 16.31 & 5.95 & 5.48 \\
\hline 92001GER & Cosmopolitan-WE & KX148135 & Germany & 1991 & Fox & 22.68 & 7.32 & 8.14 \\
\hline $86054 \mathrm{YOU}$ & Cosmopolitan-EE & KX148145 & $\mathrm{BA}$ & 1986 & Wolf & 25.54 & 7.86 & 10.11 \\
\hline 87001ARS & $\begin{array}{l}\text { Cosmopolitan- } \\
\text { ME1a }\end{array}$ & NRC-rabies IPP & Saudi Arabia & 1987 & Fox & 30.08 & 6.33 & 5.03 \\
\hline 94009TUR & Cosmopolitan-ME2 & KX148165 & Turkey & 1993 & Dog & 26.22 & 5.83 & 7.79 \\
\hline 99009BUR & ND & NRC-rabies IPP & Burma & 1999 & Dog & 31.12 & 5.97 & $\mathrm{Neg}$ \\
\hline 99008CBG & Asian-SEA3 & KX148252 & Cambodia & 1999 & Dog & 29.50 & 7.62 & 9.10 \\
\hline 99010LAO & Asian-SEA3 & KX148255 & Laos & 1999 & Dog & 31.61 & 3.77 & $\mathrm{Neg}$ \\
\hline 02043CHI & Asian-SEA2a & NRC-rabies IPP & China & ND & Dog & 29.60 & 4.02 & $\mathrm{Neg}$ \\
\hline $02045 \mathrm{CHI}$ & Asian-SEA2a & NRC-rabies IPP & China & $\mathrm{ND}$ & Dog & 36.11 & 4.02 & 7.59 \\
\hline 91014MEX & $\begin{array}{l}\text { Cosmopolitan- } \\
\text { AM2a }\end{array}$ & KX148110 & Mexico & 1991 & Dog & 30.78 & 3.35 & 6.72 \\
\hline 86001BRE & $\begin{array}{l}\text { Cosmopolitan- } \\
\text { AM3a }\end{array}$ & KX148216 & Brazil & 1986 & Dog & 28.36 & 4.33 & Neg \\
\hline 90010 NIG & Africa 2 & KX148231 & Niger & 1990 & Dog & 27.42 & 7.45 & $\mathrm{Neg}$ \\
\hline 91004USA & Arctic-related-A & KX148224 & USA & 1991 & Skunk & 23.64 & 3.93 & 7.25 \\
\hline 02052AFG & Arctic-related-AL1b & KX148225 & Afghanistan & 2002 & Dog & 22.40 & 6.84 & $\mathrm{Neg}$ \\
\hline 91041RUS & Cosmopolitan-CA1 & NRC-Rabies IPP & Russia & 1991 & Fox & 23.18 & 3.65 & 4.53 \\
\hline
\end{tabular}

Table 2. Assessment of detection spectrum of the RABV-RPA assay versus real-time RT-qPCR and the Schlottau assay. Tt: time threshold in minutes, BA: Bosnia and Herzegovina, Neg: negative; no cross-reactivity, Cq: quantitative cycle, NRC-Rabies IPD: national reference center for rabies virus (NRC-Rabies) at Institut Pasteur de Dakar, BP 220, Dakar, Senegal, NRC-Rabies IPP: national reference center for rabies virus (NRCRabies) at Institut Pasteur, Paris, France. ${ }^{a}$ Mean Cq value from duplicates with the previously described RABVL-protein real-time RT-qPCR assay by Faye et al. used as reference test ${ }^{19} .{ }^{b}$ Mean Tt value from duplicates given by the RABV-RPA assay. ${ }^{c}$ Mean Tt value from duplicates obtained with the Schlottau assay ${ }^{21}$.

an identity ranging from 98.5 to $100 \%$ to the target region of related sequences available in GenBank. Assembled sequences from the RPA amplicons were deposited in GenBank under the accession numbers MW123050-54.

Diagnostic performances. To ascertain the wide range detection capacity of the RABV-RPA assay in comparison to the Schlottau assay ${ }^{21}$ as well as the RABV-L-protein real-time RT-qPCR ${ }^{19}, 24$ RABV isolates from different parts of the world were tested in duplicate using the three methods ${ }^{40}$. In addition, a total of nineteen RABV-negative samples were also analyzed in duplicate. The RT-qPCR and the RABV-RPA assay were able to detect all positive. The median detection $\mathrm{Tt}$ of the RABV-RPA was $5.12 \pm 1.76 \mathrm{~min}$. In contrast, the Schlottau assay detected only $62.5 \%$ of analyzed isolates in a median $\mathrm{Tt}$ of $7.17 \pm 1.62 \mathrm{~min}$. The Schlottau assay gave no-fluorescence signal with nine tested isolates including those from Senegal (SA267333SEN, SA173837SEN and SH290371SEN), Burma (99009BUR), Laos (99010LAO), China (02043CHI), Brazil (86001BRE), Niger (90010NIG) and Afghanistan (02052AFG) (Table 2). All nineteen Rabies-negative samples were tested negative in all assays.

As the RT-qPCR used as reference test ${ }^{19}$, both RT-RPA assays exhibited a diagnostic specificity of $100 \%$ (95\% CI 82.35-100\%) and a positive predictive value (PPV) of 1 . However, differences were observed in other performances. The RABV-RPA showed a diagnostic sensitivity of $100 \%$ (95\% CI 85.75-100\%) and a negative predictive value (NPV) of 1 , while the Schlottau assay ${ }^{30}$ had a diagnostic sensitivity of $79.17 \%$ (95\% CI 57.85-92.87\%) a NPV of 0.7 (CI 95\% 0.63-0.89) (Fischer's exact test $\mathrm{p}<0.001$ ). A k coefficient of $1 \pm 0.15$ (95\% CI 0.70-1.29) was determined for the RABV-RPA assay, while the Schlottau assay ${ }^{21}$ showed a k coefficient of $0.77 \pm 0.14$ (95\% CI $0.47-1.06)(\mathrm{p}<0.05)$; resulting in a concordance of $100 \%$ for the RABV-RPA assay to results from the real-time RT-qPCR assay ${ }^{19}$ on the same samples (Table S1). 


\begin{tabular}{|c|c|c|c|c|c|c|c|c|}
\hline \multirow[b]{2}{*}{ TITERS ID50/mL } & \multicolumn{4}{|l|}{ RT-qPCR } & \multicolumn{2}{|c|}{ RABV-RPA } & \multicolumn{2}{|c|}{ Schlottauassay } \\
\hline & RNA molecules/reaction & Mean Cq value $^{a}$ & Mean $\mathrm{Tt}^{\mathrm{b}}$ & SD & Mean $\mathrm{Tt}^{\mathrm{b}}$ & SD & Mean $\mathrm{Tt}^{\mathrm{b}}$ & SD \\
\hline $5.2 \mathrm{E}+05$ & 32,033 & 28.42 & 57.68 & 0.480 & 3.10 & 0.020 & 5.33 & 0.030 \\
\hline $5.2 \mathrm{E}+04$ & 2374 & 31.92 & 61.71 & 0.007 & 4.36 & 0.019 & 7.83 & 0.179 \\
\hline $5.2 \mathrm{E}+03$ & 275 & 34.82 & 65.05 & 0.220 & 5.21 & 0.006 & 8.97 & 0.121 \\
\hline $5.2 \mathrm{E}+02$ & 82 & 36.45 & 66.92 & 0.672 & 5.45 & 0.086 & Neg & \\
\hline $5.2 \mathrm{E}+01$ & 11 & 39.18 & 70.06 & 0.092 & 7.49 & 0.123 & $\mathrm{Neg}$ & \\
\hline 5.2 & 8 & 39.63 & 70.58 & 0.120 & $\mathrm{Neg}$ & & $\mathrm{Neg}$ & \\
\hline $5.2 \mathrm{E}-01$ & & Neg & & & Neg & & Neg & \\
\hline $5.2 \mathrm{E}-02$ & & $\mathrm{Neg}$ & & & Neg & & Neg & \\
\hline $5.2 \mathrm{E}-03$ & & $\mathrm{Neg}$ & & & Neg & & Neg & \\
\hline $5.2 \mathrm{E}-04$ & & $\mathrm{Neg}$ & & & $\mathrm{Neg}$ & & $\mathrm{Neg}$ & \\
\hline $5.2 \mathrm{E}-05$ & & Neg & & & Neg & & Neg & \\
\hline
\end{tabular}

Table 3. Detection limit of the new established RT-RPA assay on cerebro-spinal fluid sample. Tenfold serial dilutions of RABV isolate SA217695SEN with an initial virus titer of $5.210^{6} \mathrm{ID}_{50} / \mathrm{mL}$ were tested with an rabies virus RT-RPA assay previously described by Schlottau et al. ${ }^{21}$ and the new RT-RPA assay. The corresponding numbers of RNA molecules per reaction were calculated using the mean Cq values with the standard equation previously described for the RT-qPCR used here as reference technique ${ }^{19}$. Neg: negative or no fluorescence signal, Cq : quantitative cycle number, Tt: time threshold (minutes). ${ }^{\mathrm{a}}$ Mean Cq value from duplicates. ${ }^{\mathrm{b}} \mathrm{Mean} \mathrm{Tt}$ value from duplicates.

Screening of spiked samples with the RT-RPA assays. Eleven tenfold dilutions of an archived RABV in primary cerebro-spinal fluid (CSF) were tested in duplicate with both RT-RPA assays and the realtime RT-qPCR ${ }^{19}$. The RT-qPCR was able to detect down to 8 RNA molecules/reaction within a mean Tt of $70.58 \pm 0.120 \mathrm{~min}$. However, the RABV-RPA assay detected until a titer of $11 \mathrm{RNA}$ molecules/reaction in a shorter Tt of $7.49 \pm 0.123$ min compared to the RT-qPCR. The Schlottau assay ${ }^{30}$ showed detection limit of 275 RNA molecules/reaction within an average Tt of $8.97 \pm 0.121 \mathrm{~min}$ (Table 3).

Compared to the real-time RT-qPCR ${ }^{19}$, the sensitivity of the RABV-RPA assay using serial dilutions of a CSF sample spiked with RABV was of $83.33 \%$ (95\% CI 35.88-99.58\%) with an accuracy of $90.91 \%$ (95\% CI 58.72-99.77\%), while the Schlottau assay exhibited a clinical sensitivity of 50\% (95\% CI 11.81-88.19\%) and an accuracy of 72.73\% (95\% CI 39.03-93.98\%). Our RABV-RPA assay showed agreement with real-time RT-qPCR used as reference test (Cohen's Kappa test $=0.819 ; \mathrm{p}=0.005)$ (Table S1).

In silico analysis of primers and probes sequences. Available coding-complete sequences from Laos (LAO), China (CHIN), Brazil (BRE), Niger (NIG), Afghanistan (AFG) and Senegal (SEN) enabled in silico evaluation of the Schlottau assay ${ }^{21}$ and the RABV-RPA assay using the BLAST program (https://blast.ncbi.nlm. nih.gov/).

The forward primer of the Schlottau assay (RABV-N-71Fv4) is highly similar to the target region of the isolates from Laos, China, Niger and Senegal, while it shows a dissimilarity of 5\% and $2 \%$ against the isolates from Brazil and Afghanistan, respectively. The Schlottau assay's probe (ProbeRABV-N-196-antisense) exhibits also dissimilarities of $2 \%, 4 \%, 4 \%$ and $4 \%$ to sequences from Brazil, Niger, Afghanistan and Senegal, respectively, while it is more distant to the isolates from Laos and China with dissimilarities of $10 \%$ and $16 \%$, respectively. The reverse primer of the Schlottau assay (RABV-N-211Rv1) reveals the higher dissimilarities to undetected sequences with values ranging from 41 to $50 \%$ (Table S2; Fig. 2A).

The forward primer of the RABV-RPA (RPA_N_FP2-C) is highly similar to African sequences from Senegal and Niger with distances ranging from 0 to 3\% and 3\%, respectively, while it shows dissimilarities of $11 \%$ to the isolates from Laos, China, and Afghanistan. However, this primer exhibits a dissimilarity of $17 \%$ to the sequence from Brazil. The probe of the RABV-RPA (exoprobe-N) also is highly similar to the target region of the isolate from Niger and shows a dissimilarity to the Senegalese sequences between 4 and $6 \%$. However, it is distant to the isolates from Brazil, China, Laos and Afghanistan with dissimilarities of $8 \%, 8 \%, 13 \%$ and $17 \%$, respectively. As the corresponding forward primer, the reverse primer of the RABV-RPA is higher similar to the African sequences. However, it exhibits dissimilarities of $6 \%, 16 \%, 16 \%$ and $19 \%$ to the isolates from Brazil, China, Afghanistan and Laos, respectively (Table S2; Fig. 2B). Nevertheless, these dissimilarities didn't omit detection of the aligned sequences by the RABV-RPA assay (Fig. 2B).

\section{Discussion}

Despite being preventable, rabies is responsible for thousands of human deaths worldwide each year, mostly in Africa and Asia ${ }^{23,41}$. Many rabies endemic areas don't have the resources to implement Direct Fluorescent Antibody testing, which also need a fluorescent microscope and highly trained technicians ${ }^{16}$. On the top of that, a continuous cold storage of samples is a big limiting factor. Therefore, according to the WHO orientations related to the need for rapid and economical diagnosis tests ${ }^{23}$, considerable efforts are made to develop accessible direct detection methods based on RT-qPCR, which allow a rapid and highly sensitive detection of RABV. Nevertheless, 


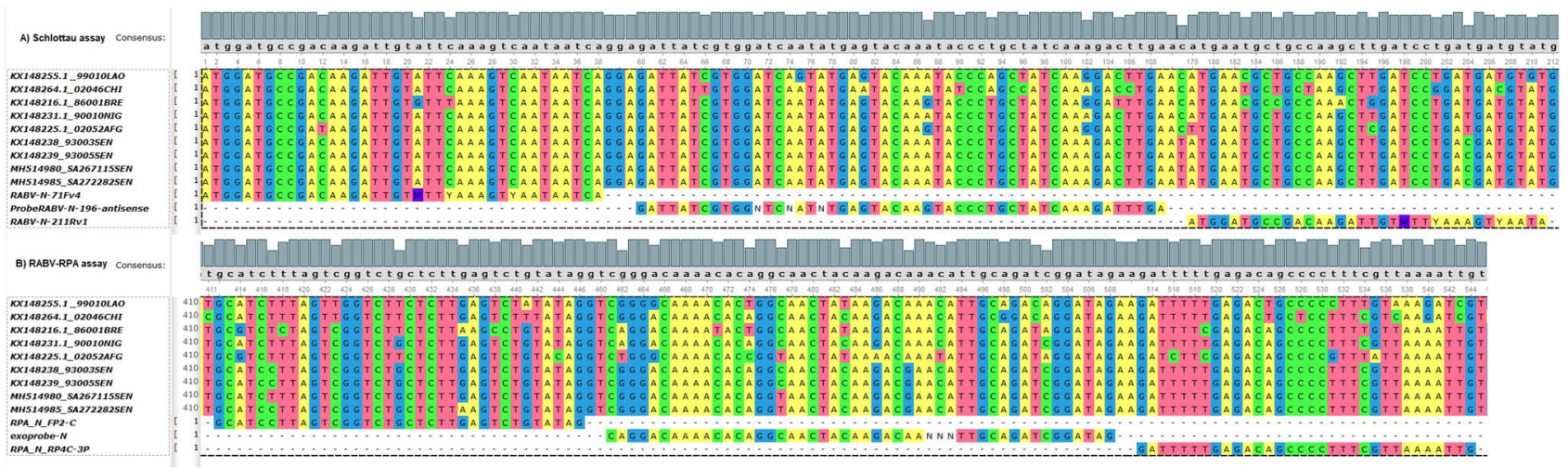

Figure 2. In silico analysis of (A) the Schlottau assay and (B) the RABV-RPA assay again sequences of differences rabies virus isolates from Laos (LAO), China (CHIN), Brazil (BRE), Niger (NIG), Afghanistan (AFG) and Senegal (SEN), respectively. The alignment was performed using the MAFFT algorithm implemented in the Unipro UGENE software ${ }^{50}$. The in silico analysis revealed signature erosion in the reverse primer of the Schlottau assay, indicating that this rabies virus diagnostic method have issues with sensitivity when used to detect some isolates from these countries.

these technologies require the use of complex instruments and well-equipped laboratories ${ }^{17-19,22}$. A field-friendly assay would enable transmission control in low resource settings and help in surveillance under field condition.

In this study, we developed and validated a reliable and highly sensitive RT-RPA assay for RABV detection. The assay could be easily implemented with minimal equipment and training at remote areas, where the real burden of the disease is underestimated ${ }^{41}$.

Since a program or strict rules are not available, the most challenging step in the RABV-RPA assay development is the design of a primer pair able to amplify a very low RNA copy number ${ }^{31,42,43}$. Nevertheless, a highly sensitive RT-RPA for rapid detection of RABV was successfully established, exhibiting a reliable detection until 4 RNA molecules/reaction within $10 \mathrm{~min}$. The RABV-RPA displayed only a $1-\log _{10}$-step reduction compared to the reference standard RT-qPCR assay ${ }^{19}$ and is more sensitive than the Schlottau assay (1000 RNA molecules/ reaction $)^{21}$ and a recently described RT-RPA assay (562 RNA molecules/reaction) ${ }^{44}$. In addition, obtaining results between 2 to 10 min demonstrated that the RABV-RPA assay is also much faster than the previously described rabies virus isothermal molecular tests ${ }^{21,45-48}$ without affecting the sensitivity.

Despite few mismatches, the RABV-RPA assay shows better detection performances on isolates from various regions of the world than the Schlottau assay ${ }^{21}$, which was less sensitive for isolates from Senegal, Burma, Laos, China, Niger, Brazil and Afghanistan. This might be related to signature erosion in it reverse primer. Therefore, our RT-RPA method is proposed as suitable for rabies diagnostic in the field, particularly in low-resource areas in West-Africa, South-Asia, South-America and Middle-East where rabies still circulating.

In contrast to the Schlottau assay, the RABV-RPA assay showed high accuracy on viral dilutions in a nonneural clinical specimen (CSF) and displayed good agreement with the RT-qPCR ${ }^{19}$. Based on its performances, our RABV-RPA presented here could be useful as reliable point-of-need tool for ante-mortem rabies diagnosis in humans, as well as a valuable surveillance tool for rapid local decision-making. Furthermore, an outstanding advantage for the RT-RPA method is that could be used with a relatively basic portable heat-source, and therefore making the RABV-RPA a potential point-of-need alternative for RABV detection in resource-limited settings. Finally, our RABV-RPA assay, could be a complement for existing methods for rabies diagnosis ${ }^{19,21,22}$ with a high specificity, sensitivity and repeatability and more suitable for rapid and broad detection of RABV isolates.

In conclusion, the RABV-RPA method developed in this study demonstrates high sensitivity and specificity for detection of RABV. In addition, a validation experiment showed its clinical applicability and could be used for rapid and accurate ante-mortem diagnosis in human. The RABV-RPA could be used in a suitcase laboratory with rapid extraction methods which had previously shown best performances in extraction of rabies virus RNA $^{21,31,49,50}$ and easily implemented in routine laboratory activities as a rapid confirmatory test to first-line assays or in low-resource settings by including portable heat-sources with solar-powered battery ${ }^{34}$.

\section{Methods}

Ethical statement. All samples used in this study were collected in the frame of the national integrated surveillance program for Rabies in Senegal or available from routine diagnostic activities of the national reference centers for rabies diagnosis in Senegal and Paris.

According to IACUC animal guidelines ${ }^{51}$, suckling mice were used in routine virus isolation at the animal laboratory in Institute Pasteur in Dakar accredited by WHO as Collaborating Centre for Arboviruses and Hemorrhagic Fevers, for surveillance, diagnostics and research as approved by the Senegalese national ethical committee. All viral isolations in suckling mice were performed in accordance with the ARRIVE guidelines ${ }^{52}$.

Design of RABV-RPA primers and exo probe. The RABV-RPA primers were designed by in silico multiple alignments of available Africa 1 and Africa 2 RABV sequences from GenBank using the Muscle algorithm 


\begin{tabular}{|c|c|c|c|c|c|c|c|}
\hline Name & Type & Length (bp) & Sense & Sequence $5^{\prime}-3^{\prime}$ & Gene & Position $^{\mathrm{a}}$ & Product size (bp) \\
\hline RPA_N_FP2C & Primer & 35 & Forward & GCATCCTTAGTCGGTCTGCTCTTGAGTCTGTATAG & $\mathrm{N}$ & $482-516$ & \multirow{3}{*}{133} \\
\hline Exo_probe_N & Probe & 45 & Reverse & $\begin{array}{l}\text { CTATCCGATCTGCAA[BHQ-dT][THF][FAM-dT]TTGTCTTGTAGTTGC } \\
\text { CTGTGTTTTGTCCTG-Ph }\end{array}$ & $\mathrm{N}$ & $531-578$ & \\
\hline RPA_N_RP4C3P & Primer & 32 & Reverse & CAATTTTAACGAAAGGGGCTGTCTCAAAAATC & $\mathrm{N}$ & $583-614$ & \\
\hline
\end{tabular}

Table 4. Oligonucleotide sequences of primers and probe designed for the RABV-RPA assay. FAM: fluorescein amidit; BHQ: blackberry quencher; THF: tetrahydrofuran; Ph: phosphate; bp: base-pairs. ${ }^{a}$ Corresponding nucleotide positions on genome of the rabies virus strain from Nigeria DRV-NG11(GenBank Ac. No. KC196743).

implemented in the Unipro UGENE software ${ }^{53}$. According to RPA guidelines from TwistDx (Cambridge, UK), a total of seventeen forward primers (FPs), twenty seven reverse primers (RPs), and one fluorescent exo probe were designed based on the conserved region of the nucleocapsid gene $(\mathrm{N})$. The exo-probe was designed with an inverse arrangement of fluorophore (6-carboxyfluorescein [FAM]), quencher (black hole quencher 1 [BHQ-1]), internal abasic site mimic (tetrahydrofuran spacer [THF]) and block elongation (phosphate [P]). All oligonucleotides were produced by TIB MolBiol (Berlin, Germany). All 179 primer combinations were tested to select the RPA primers and probe set yielding the highest analytical sensitivity using the $10^{5}$ RNA in vitro transcripted molecular standard ordered from Genexpress (Berlin, Germany) (Table 4).

RNA extraction and samples preparation. All thirty seven virus isolates analyzed in this study were previously cultured on suckling mice brain and derived from collection of national reference center for rabies diagnosis in Senegal at Institut Pasteur de Dakar (NRC-Rabies IPD) (Tables 1, 2). A total of 17 RNA extracts from primary rabies-positive brain samples representative of the existing RABV diversity were provided by the national reference center for rabies diagnosis at Institut Pasteur of Paris, France (NRC-Rabies IPP) (Table 2). The CSF sample was obtained from the collection of the laboratory for medical analysis at IPD. Extraction was carried out in the Biosafety level 3 (BSL3) containment facilities at Institut Pasteur de Dakar respecting the WHO's biosafety guidelines and requirements for conducting work on infectious microorganisms and other biological hazards such as RABV ${ }^{54}$.

Extraction of viral RNA from $140 \mu \mathrm{L}$ of mice brain and tenfold serial dilutions of a CSF sample was performed with the QIAamp viral RNA mini kit (Qiagen, Heiden, Germany) according to manufacturer's instructions and eluted in a final volume of $50 \mu \mathrm{L}$. Extracted RNA was frozen at $-80^{\circ} \mathrm{C}$ prior to downstream applications.

Rabies virus RT-RPA assay. RABV-RPA amplifications were achieved in a $50 \mu \mathrm{L}$ volume using the TwistAmp RT exo kits (TwistDx, Cambridge, UK). For each reaction, $29.5 \mu \mathrm{l}$ of rehydration buffer, $420 \mathrm{nM}$ RPA primers, $120 \mathrm{nM}$ exo probe and $6.7 \mu \mathrm{l}$ of nuclease-free water were added into the lids. A $5 \mu \mathrm{L}$ of viral RNA diluted $1 / 10$ in nuclease-free water and $14 \mathrm{mM}$ final concentration of magnesium acetate were subsequently added into each tube lid. Then the lids were closed carefully and the reaction mix was centrifuged into the lyophilized reaction pellet containing a dried enzyme. The mix was vortexed and spun down once again, and the tubes were immediately placed in the Twista Tubescanner device (TwistDx, Cambridge, UK) connected to a computer for real-time monitoring of fluorescence signal. The reaction was performed at $42{ }^{\circ} \mathrm{C}$ for 15 min, with brief mixing and centrifugation of reaction tubes after $230 \mathrm{~s}$ of the incubation in order to improve the sensitivity of the assay. Real-time detection of RT-RPA amplicons relies on the exonuclease enzyme, which cleaves at the THF site between fluorophore and quencher in the exo-probe and the generated fluorescence signal is measured each $10 \mathrm{~s}$ using the FAM channel. The resulting amplification curves were analyzed by Twista Studio software using a combined threshold and first derivation analysis for signal interpretation. Samples producing an exponential amplification curve above the threshold of the negative control were considered positive.

Analytical specificity. RNA extracts from 15 RABV-positive isolates and 15 isolates of other rhabdoviruses genetically related to RABV were tested in duplicates in order to evaluate the specificity of the RABV-RPA assay (Table 1). Positive and negative controls containing standard RNA and nuclease-free water were included in each run. The real-time RT-qPCR assay was used as reference test and was performed using the Quantitect Probe RT-PCR Kit (Qiagen, Heiden, Germany) in a final volume of $25 \mu$ following previously published protocol ${ }^{19}$. Amplicons obtained with the RABV-RPA assay from five isolates from Niger (90010NIG), Brazil (86001BRE), Afghanistan (02052AFG), Cambodia (99008CBG) and Saudi Arabia (87001ARS) (Table 2), were purified using AmPure magnetic beads (Agencourt Bioscience [Beckman Coulter Inc.], Beverly, MA, USA), and adapters were ligated using the Nextera XT library Prep kit according to the manufacturer's recommendations (Illumina, San Diego, CA, USA). The purified and amplified libraries were quantified using the KAPA library quantification kit (Kapa Biosystems, Wilmington, DE, USA) and subsequently sequenced on the Illumina MiSeq platform with 76 bp paired-end reads using the Miseq reagents kit v3 according to the manufacturer's protocol (Illumina, San Diego, CA, USA). Reads were quality trimmed using Prinseq-lite, and de novo genome assembly was performed with IDBA (Iterative de Bruijn graph De Novo Assembler). The coverage and the identity of obtained sequences to the target region were verified using a BLAST analysis (https://blast.ncbi.nlm.nih.gov/). 
Analytical sensitivity. Analytical sensitivity of the RABV-RPA assay was assessed using eight datasets from tenfold dilutions of the in vitro standard RNA produced by Genexpress (Berlin, Germany) ranging from $10^{7}$ to 1 molecules/reaction. The RT-qPCR was used as reference method ${ }^{19}$. The Tt data values are provided as the mean and standard deviation (SD). In addition, the inter-assay and intra-assay repeatability were determined by CVs from $8 \mathrm{Tt}$ values of the dilution $10^{5}$ standard RNA molecules/reaction in 8 different runs and in the same run, respectively.

Diagnostic performances. In order to assess its reliability and efficiency, a collection of twenty-four positive specimens from different countries of the world ${ }^{40}$ was tested in duplicate with both the RABV-RPA assay and the Schlottau assay ${ }^{21}$ as well as the real-time RT-qPCR assay as reference test ${ }^{19}$. Nineteen Rabies-negative samples were also used with both assays for assessment of diagnostic specificity. PPV and NPV, diagnostic sensitivity and specificity were calculated using standard formulas ${ }^{19}$.

Experimental comparison of the RT-RPA assays. To assess the usefulness of the RABV-RPA assay for ante-mortem diagnosis of rabies in human, tenfold serial dilutions from a positive RABV stock with a concentration of 32,033 RNA molecules/reaction (titer of $5.210^{6} \mathrm{ID}_{50} / \mathrm{mL}$ ) were performed in a CSF sample tested negative for Streptococcus, Salmonella, Legionella, Leptospirosis, Borellia, Listeria, Mycoplasma and viral herpes panel (data not shown). RNA extracts were tested in duplicate with both assays in order to determine the limit of detection (LOD) in the presence of sample background. The LOD was determined in copy/ reaction using cycle quantitative $(\mathrm{Cq})$ values and the standard equation from the real-time RT-qPCR assay used as reference test ${ }^{19}$.

Assessment of the impact of genetic diversity on RT-RPA assays. In silico analysis of both RTRPA assays was performed again sequences of rabies virus isolates that were not detected during the sensitivity assessment by the Schlottau assay ${ }^{21}$, using the MAFFT alignment algorithm implemented in the Unipro UGENE software $e^{53}$.

Statistical methods. A semi-log regression analysis and a probit analysis were performed by plotting the RABV-RPA Tt values against the number of molecules detected in eight replicates of tenfold dilutions of the in vitro standard RNA ranging from $10^{7}$ to 1 molecules/reaction (8/8) using PRISM (Graphpad Software Inc., San Diego, California) and STATISTICA (StatSoft, Hamburg, Germany), respectively. We also used a kappa test to compare diagnostic performances from the RABV-RPA assay and the Schlottau assay ${ }^{21}$ to results of the RT-qPCR assay ${ }^{19}$ where the Cohen's kappa coefficient $(k)$ represents a measure of the agreement between two assays. A p-value $(\mathrm{p})<0.05$ was considered as statistically significant. In addition, sensitivity and accuracy of both RT-RPA assays were determined with a $95 \%$ confidence interval and their concordance to the reference test was analyzed using Cohen's kappa test and considering a $\mathrm{p}<0.05$ as significant.

Received: 1 May 2020; Accepted: 13 January 2021

Published online: 04 February 2021

\section{References}

1. Delmas, O. et al. Genomic diversity and evolution of the lyssaviruses. PLoS ONE 3(4), e2057 (2008).

2. Bourhy, H. et al. The origin and phylogeography of dog rabies virus. J. Gen. Virol. 89, 2673-2681 (2008).

3. Talbi, C. et al. Evolutionary history and dynamics of dog rabies virus in western and central Africa. J. Gen. Virol. 90(Pt 4), 783-791 (2009).

4. Hampson, K. et al. Estimating the global burden of endemic canine rabies. PLoS Negl. Trop. Dis. 9(4), e0003709 (2015).

5. Dietzschold, B. et al. Pathogenesis of rabies. Curr. Top. Microbiol. Immunol. 292, 45-56 (2005).

6. Ross, R. S. et al. Instructive even after a decade: Complete results of initial virological diagnostics and re-evaluation of molecular data in the German rabies virus "outbreak" caused by transplantations. Int. J. Med. Microbiol. 305(7), 636-643 (2015).

7. Vora, N. M. et al. Transplant-Associated Rabies Virus Transmission Investigation Team. Raccoon rabies virus variant transmission through solid organ transplantation. JAMA 310(4), 398-407 (2013).

8. Dacheux, L. et al. More accurate insight into the incidence of human rabies in developing countries through validated laboratory techniques. PLoS Negl. Trop. Dis. 4(11), e765 (2010).

9. World Health Organization. Expert Consultation on Rabies, Second report. World Health Organ. Tech. Rep. Ser. 982, 1-150 (2013).

10. Madhusudana, S. N. et al. Evaluation of a direct rapid immunohistochemical test (dRIT) for rapid diagnosis of rabies in animals and humans. Virologica Sinica 27(5), 299-302 (2012).

11. Stein, L. T. et al. Immunohistochemical study of rabies virus within the central nervous system of domestic and wildlife species. Vet. Pathol. 47(4), 630-633 (2010).

12. Servat, A. et al. Evaluation of a rapid immunochromatographic diagnostic test for the detection of rabies from brain material of European mammals. Biologicals 40(1), 61-66 (2012).

13. Kasempimolporn, S. et al. Evaluation of a rapid immunochromatographic test strip for detection of rabies virus in dog saliva samples. J. Vet. Diagn. Investig. 23(6), 1197-1201 (2011).

14. World Organization for Animal Health. Manual of diagnostic tests and vaccines for terrestrial animals, 6th ed. Paris http://www. oie.int/international-standard-setting/terrestrial-manual (2011).

15. Rudd, R. J. et al. A need for standardized rabies-virus diagnostic procedures: Effect of cover-glass mountant on the reliability of antigen detection by the fluorescent antibody test. Virus Res. 111(1), 83-88 (2005).

16. Dacheux, L. et al. More accurate insight into the incidence of human rabies in developing countries through validated laboratory techniques. PLoS Negl. Trop. Dis 4(11), e765 (2010).

17. Wacharapluesadee, S. et al. Detection of rabies viral RNA by TaqMan real-time RT-PCR using non-neural specimens from dogs infected with rabies virus. J. Virol Methods 184(12), 109-112 (2012). 
18. Dacheux, L. et al. Dual combined real-time reverse transcription polymerase chain reaction assay for the diagnosis of lyssavirus infection. PLoS Negl. Trop. Dis. 10(7), e0004812 (2016).

19. Faye, M. et al. Development and validation of sensitive real-time RT-PCR assay for broad detection of rabies virus. J. Virol. Methods 243, 120-130 (2017).

20. Mani, R. S. et al. Utility of real-time Taqman PCR for antemortem and postmortem diagnosis of human rabies. J. Med. Virol. 86(10), 1804-1812 (2014).

21. Schlottau, K. et al. Development of molecular confirmation tools for swift and easy rabies diagnostics. Virol. J. 14, 184 (2017).

22. Gigante, M. C. et al. Multi-site evaluation of the LN34 pan-lyssavirus real-time RT-PCR assay for post-mortem rabies diagnostics. PLoS ONE 13(5), e0197074 (2018).

23. Banyard, A. C. et al. Control and prevention of canine rabies: The need for building laboratory-based surveillance capacity. Antiviral Res. 98(3), 357-364 (2013).

24. Yan, L. et al. Isothermal amplified detection of DNA and RNA. Mol. Biosyst. 10, 970-1003 (2014).

25. Daher, R. K. et al. Recombinase polymerase amplification for diagnostic applications. Clin. Chem. 62(7), 947-958 (2016).

26. de Paz, H. D. et al. Molecular isothermal techniques for combating infectious diseases: Towards low-cost point-of-care diagnostics. Expert Rev. Mol. Diagn. 14, 827-843 (2014).

27. Giuffrida, M. C. \& Spoto, G. Integration of isothermal amplification methods in microfluidic devices: Recent advances. Biosens. Bioelectron. 90, 174-186 (2017).

28. Amer, H. M. et al. A new approach for diagnosis of bovine coronavirus using a reverse transcription recombinase polymerase amplification assay. J. Virol. Methods 193, 337-340 (2013).

29. Armes, N. A. \& Stemple, D. L. Recombinase Polymerase Amplification. US Patent, Vol. 7, 428-485 (2009).

30. Piepenburg, O. et al. DNA detection using recombination proteins. PLoS Biol. 4, e204 (2006).

31. Abd El Wahed, A., Weidmann, M. \& Hufert, F. T. Diagnostics-in-a-suitcase: development of a portable and rapid assay for the detection of the emerging avian influenza A (H7N9) virus. J. Clin. Virol. 69, 16-21 (2015).

32. Kersting, S. et al. Rapid detection of Plasmodium falciparum with isothermal recombinase polymerase amplification and lateral flow analysis. Malar J. 13, 99 (2014).

33. Euler, M. et al. Development of a panel of recombinase polymerase amplification assays for the detection of biothreat agents. J. Clin. Microbiol. 51, 1110-1117 (2013).

34. Boyle, D. S. et al. Rapid detection of Mycobacterium tuberculosis by recombinase polymerase amplification. PLoS ONE 9, e103091 (2014).

35. Abd El Wahed, A. et al. A portable reverse transcription recombinase polymerase amplification assay for rapid detection of footand-mouth disease virus. PLOS ONE 8, e71642 (2013).

36. Teoh, B. T. et al. Early detection of dengue virus by use of reverse transcription-recombinase polymerase amplification. J. Clin. Microbiol. 53, 830-837 (2015).

37. Abd El Wahed, A. et al. Recombinase polymerase amplification assay for rapid diagnostics of dengue infection. PLoS ONE 10, e0129682 (2015).

38. Zhang, S. et al. Rapid diagnostic detection of plum pox virus in Prunus plants by isothermal AmplifyRP" using reverse transcriptionrecombinase polymerase amplification. J. Virol. Methods 207C, 114-120 (2014).

39. Robardet, E. et al. International interlaboratory trials on rabies diagnosis: An overview of results and variation in reference diagnosis techniques (fluorescent antibody test, rabies tissue culture infection test, mouse inoculation test) and molecular biology techniques. J. Virol. Methods 177, 15-25 (2011).

40. Troupin, C. et al. Large-scale phylogenomic analysis reveals the complex evolutionary history of rabies virus in multiple carnivore hosts. PLoS Pathog. 12(12), e1006041 (2016).

41. Hampson, K. et al. Estimating the global burden of endemic canine rabies. PLoS Negl. Trop. Dis. 9, e0003709 (2015).

42. Faye, O. et al. Development and deployment of a rapid recombinase polymerase amplification Ebola virus detection assay in Guinea in 2015. Euro Surveill. 20(44), 1560-7917 (2015).

43. Euler, M. et al. Development of a panel of recombinase polymerase amplification assays for detection of biothreat agents. J. Clin. Microbiol. 51(4), 1110-1117 (2013).

44. Coertse, J. et al. Reverse transcription recombinase polymerase amplification assay for rapid detection of canine associated rabies virus in Africa. PLoS ONE 14(7), e0219292 (2019).

45. Boldbaatar, B. et al. Rapid detection of rabies virus by reverse transcription loop-mediated isothermal amplification. Jpn. J. Infect. Dis. 62(3), 187-191 (2009).

46. Saitou, Y. et al. A method for simultaneous detection and identification of Brazilian dog- and vampire bat-related rabies virus by reverse transcription loop-mediated isothermal amplification assay. J. Virol. Methods 168(1-2), 13-17 (2010).

47. Wacharapluesadee, S. et al. Comparative detection of rabies RNA by NASBA, real-time PCR and conventional PCR. J. Virol. Methods 175(2), 278-282 (2011).

48. Muleya, W. et al. Molecular epidemiology and a loop-mediated isothermal amplification method for diagnosis of infection with rabies virus in Zambia. Virus Res. 163(1), 160-168 (2012).

49. Mondal, D. et al. Mobile suitcase laboratory for rapid detection of Leishmania donovani using recombinase polymerase amplification assay. Parasites Vectors 9, 281 (2016).

50. Weidmann, M. et al. Development of mobile laboratory for viral haemorrhagic fever detection in Africa. J. Infect. Dis. 218, $1622-1630$ (2018).

51. University of Iowa Principles for the Care and Use of Laboratory Animals (IACUC). AVMA Guidelines for the Euthanasia of Animals: 2013 Edition, AVMA.org. (2013), Updated 1/11/17.

52. Percie du Sert, N. et al. The ARRIVE guidelines 2.0: Updated guidelines for reporting animal research. PLoS Biol. 18(7), e3000410 (2020).

53. Okonechnikov, K., Golosova, O. \& Fursov, M. The UGENE team. Unipro UGENE: A unified bioinformatics toolkit. Bioinformatics 28, 1166-1167 (2012).

54. World Health Organization. Laboratory biosafety manual, 3rd ed. World Health Organization. https://apps.who.int/iris/handl e/10665/42981. (2004)

\section{Acknowledgements}

We acknowledge all colleagues of the National Reference Centre for Rabies, virology department of Institut Pasteur de Dakar, Senegal for sharing virus stocks and supportive information necessary for establishment and accomplishment of this study. We also thank colleagues from the Institut Pasteur, Lyssavirus Dynamics and Host Adaptation Unit, National Reference Centre for Rabies, WHO Collaborating Center for Reference and Research on Rabies, Paris, France for sharing RNA extracts. 


\section{Author contributions}

Conceived and designed the experiments: M.F., A.A.E.W., A.A.S. and O.F.; Performed the experiments: M.F., and A.A.E.W.; Analyzed the data: M.F., A.A.E.W., O.F. and J.K.; Contributed for reagents/materials: A.A.E.W., B.H., A.A.S., O.F. and O.F.; Wrote the paper: all the authors. First author: MF.

\section{Funding}

This research received no specific grant from any funding agency in the public, commercial, or not-for-profit sectors and was only supported by the Institut Pasteur de Dakar internal funds for research.

\section{Competing interests}

The authors declare no competing interests.

\section{Additional information}

Supplementary Information The online version contains supplementary material available at https://doi. org/10.1038/s41598-021-82479-8.

Correspondence and requests for materials should be addressed to M.F.

Reprints and permissions information is available at www.nature.com/reprints.

Publisher's note Springer Nature remains neutral with regard to jurisdictional claims in published maps and institutional affiliations.

(c) (i) Open Access This article is licensed under a Creative Commons Attribution 4.0 International License, which permits use, sharing, adaptation, distribution and reproduction in any medium or format, as long as you give appropriate credit to the original author(s) and the source, provide a link to the Creative Commons licence, and indicate if changes were made. The images or other third party material in this article are included in the article's Creative Commons licence, unless indicated otherwise in a credit line to the material. If material is not included in the article's Creative Commons licence and your intended use is not permitted by statutory regulation or exceeds the permitted use, you will need to obtain permission directly from the copyright holder. To view a copy of this licence, visit http://creativecommons.org/licenses/by/4.0/.

(C) The Author(s) 2021 